Conclusion* There was no difference in oncologic outcomes when comparing minimally invasive and open surgery among high-risk endometrial cancer patients

\section{EFFECTIVENESS AND SAFETY OF SENTINEL NODE IN ENDOMETRIAL CANCER : REVIEW OF THE LITERATURE}

${ }^{1} \mathrm{~S}$ Petousis ${ }^{*},{ }^{2} \mathrm{C}$ Margioula Siarkou, ${ }^{2} \mathrm{~A}$ Papanikolaou, ${ }^{2} \mathrm{~K}$ Dinas, ${ }^{2} \mathrm{~A}$ Daniilidis. ${ }^{1}$ Aristotle University of Thessaloniki; ${ }^{2}$ Aristotle University of Thessaloniki, 2nd Department of Obstetrics and Gynaecology, Thessaloniki, Greece

\subsection{6/ijgc-2021-ESGO.207}

Introduction/Background* Main objective of the present study is to discuss the effectiveness and safety of sentinel lymph node in endometrial cancer cases.

Methodology A comprehensive review of published literature in Pubmed, with special focus on meta-analyses and prospective studies, was performed.

Result(s)* Sentinel lymph node (SLN) is indicated for treatment of low and intermediate risk endometrial cancer. Since lymphadenectomy is an important source of morbidity $(17.5 \%)$ with $2.5 \%$ mortality and no proven therapeutic value has been indicated for systematic lymphadenectomy, minimization of approach aims to reduce postoperative morbidity such as lymphedemas. Blood loss, operation time and postoperative complications of SLN are comparable with no lymphadenectomy and significantly decrease compared with systematic lymphadenectomy. SLN detection rates are reported to reach $97 \%$ per patient, $87 \%$ per hemipelvis and $78 \%$ bilaterally. SLNs detected bilaterally are associated with $95.8 \%$ sensitivity and over than $98 \%$ negative predictive value. Use of SLN strategy is proven to mitigate concern for missed paraaortic micormetastasis, thereafter eliminating risk for postoperative overtreatment. Finally, regarding used regimen, ICG is potentially superior to blue dye as its use was associated with $26.5 \%$ increase od SLN dtections rates. Furthermore, a larger dose of ICG is associated with a higher number of retrieved SLNs but not with an increased bilateral DR

Conclusion* SLN is a safe and effective strategy to identify nodal micrometastasis, thereafter optimizing complentary therapy in low and intermediate risk endometrial cancer patients.

\section{BLOODLESS MANAGEMENT OF PATIENT UNDERGOING PELVIC EXENTERATION}

${ }^{1} \mathrm{H}$ Jiang ${ }^{*},{ }^{2} V$ Le Thanh, ${ }^{3} \mathrm{C}$ Pappa, ${ }^{3} \mathrm{M}$ Abdalla, ${ }^{3} \mathrm{~S}$ Smyth, ${ }^{1} \mathrm{H}$ Soleymani Majd. ${ }^{1}$ Churchill Hospital, Gynaeoncology, UK; ${ }^{2}$ John Radcliffe Hospital, UK; ${ }^{3}$ Churchill Hospital, UK

\subsection{6/ijgc-2021-ESG0.208}

Introduction/Background* Blood transfusions are common in the surgical management of gynaecology oncology patients, up to $93 \%$ of patients undergoing pelvic exenteration may require blood products. However, increasingly more patients are cautious in receiving blood products, either for fear of potential risks or for religious believes. It is therefore vital to optimize the management of these patients in order to avoid blood transfusions.

We describe the case of a 58-year old female Jehovah's witness patient undergoing pelvic exenteration, focusing on the preoperative, intraoperative and postoperative measures that allowed an uncomplicated surgery without blood transfusion.
Methodology In this case, we summarize the management of a 58-year old lady who underwent laparotomy, pelvic exenteration, Bricker colicureterostomy, end colostomy formation for recurrent endometrial carcinoma, despite previous total abdominal hysterectomy and bilateral salpingo-oophorectomy followed by brachytherapy, chemotherapy and external beam radiotherapy for high grade serous carcinoma.

Result(s)* Preoperatively, an advance decision to refuse blood products was discussed, to ascertain all the options that were suitable. Since her preoperative haemoglobin was acceptable $(127 \mathrm{~g} / \mathrm{L})$, no further intervention was required. Intraoperatively, blood loss was effectively minimised with meticulous haemostasis, intraoperative haemodilution and cell salvage. Despite these interventions, total blood loss was $1030 \mathrm{~mL}$ and postoperative haemoglobin was $113 \mathrm{~g} / \mathrm{L}$. Postoperative measures therefore included intravenous iron infusion, minimisation of phlebotomy and optimisation of cardiopulmonary status. Erythropoietin was also considered, but was not necessary as patient responded to the previous measures well and was successfully discharged after an uncomplicated recovery.

Conclusion* Only a few cases of total pelvic exenteration have been described in the literature for Jehovah's witness patients. However, our case shows that laparotomy and pelvic exenteration is feasible in patients refusing blood products, if performed under a multidisciplinary team and with careful preoperative, intraoperative and postoperative planning, also in the setting of previous radical hysterectomy and co-adjuvant therapy.

\section{ECTOPIC LEFT RENAL ARTERY ORIGINATING FROM LEFT COMMON ILIAC: A RARE ANATOMIC VARIATION}

S Petousis*, C Margioula-Siarkou, A Daniilidis, G Mavromatidis, A Papanikolaou, K Dinas. 2nd Department of Obstetrics and Gynaecology

\subsection{6/ijgc-2021-ESG0.209}

Introduction/Background* Main objective is to present the case of a rare anatomic variation observed during laparotomy procedure along with performing a relative review of the literature.

Methodology Medical eleents of patient were reviewed with special focus on epidemiological, surgical and histopathological characteristics. A comprehensive review of published literature in Pubmed, with special focus on meta-analyses and prospective studies, was also performed.

Result(s)* A 56-year old woman was operated with laparotomy to peform full surgical staging with total hysterectomy with bilateral salpingoophorectomy, pelvic lymphadenectomy, paraaortic lymphadenectomy and infracolic omentectomy because of initial diagnosis of serous endometrial cancer. During laparotomy procedure, the diagnosis of an ectopic left renal artery (LRA) originating directly from the left common iliac artery (LCIA) was made. Woman was already known to have an ectopic pelvic kidney (EPK). EPK was found in retroperitoneal space, approximately in the level of sigmoidal bend. The LRA indeed originated just $2 \mathrm{~cm}$ below the level of bifurcation, while the left renal vein (LRV) was originated relatively from the left iliac vein (LIV) also $2-3 \mathrm{~cm}$ below the level of venal bifurcation, having a parallel route just below the LRA.

To our knowledge, this is the first published case of such an anatomical intraoperative finding, which indicates the high complexity degree that may characterize the performance of a para-aortic lymphadenectomy. 


\section{Correction: 872 bloodless management of patient undergoing pelvic exenteration}

Jiang H, Le Thanh V, Pappa C, et al. 872 Bloodless management of patient undergoing pelvic exenteration. Int J Gynecol Cancer 2021;31:A129. doi: 10.1136/ijgc-2021-ESG0.208

This Abstract has been corrected since it was first published. In the Results section, postoperative hemoglobin has been updated from $66 \mathrm{~g} / \mathrm{L}$ to $113 \mathrm{~g} / \mathrm{L}$.

(C) IGCS and ESG0 2022. No commercial re-use. See rights and permissions. Published by BMJ.

Int J Gynecol Cancer 2022;32:115. doi:10.1136/ijgc-2021-ESG0.208corr1

A) Check for updates 